\title{
Intensity-induced quenching of absorption of diatomic molecules in two near-resonance laser fields
}

\author{
Munir H. Nayfeh, Kevin King, G. B. Hillard, and I. S. Shahin* \\ Department of Physics, 1110 West Green Street, University of Illinois at Urbana-Champaign, Urbana, Illinois 61801 \\ Ali H. Nayfeh ${ }^{\dagger}$ \\ Virginia Polytechnic Institute and State University, Blacksburg, Virginia 24061
}

(Received 1 March 1982)

\begin{abstract}
The interaction of a diatomic molecule with two near-resonance laser fields is investigated with the use of a one-dimensional anharmonic oscillator model. Our study shows that, over a certain range of intensity of one of the laser fields, induced quenching of the absorption takes place.
\end{abstract}

The interaction of laser beams with molecular systems has recently received considerable attention. ${ }^{1,2}$ The interest stems from a number of important applications which include laser isotope separation, laser control of chemical reactions, enhancing radiative collisional transfer, and collisional ionization in atomic and molecular collisions.

Recently, a model which involves two types of processes was suggested to explain a threshold behavior of multiphoton dissociation of molecules induced by an intense resonant mode. ${ }^{3,4}$ The first process is a resonant excitation at low vibrational transitions in a field of moderate intensity; the second process involves nonresonant excitation at transitions in vibrational quasicontinuum under a sufficiently intense field. ${ }^{3,5,6}$ The model was checked experimentally. However, the experiments ${ }^{4}$ showed that the width of the resonant absorption is much larger than what is expected.

Excitation with multimode radiation sources has recently received increased theoretical attention. ${ }^{7-10}$ Classical mechanics was used to show that hydrogen flouride may be more easily dissociated with two infrared lasers of different frequencies than with just one. ${ }^{7}$ It was also shown that the two-laser dissociation proceeds by a qualitatively different process than that of the one-laser dissocation. ${ }^{7}$ The effect of laser polarization on the two-mode interaction was also investigated. ${ }^{9.10}$ It was found that the excitation is more effective when the polarizations of the two modes are parallel than when they are perpendicular.

In view of the above interest, we analyze in this paper the response of a diatomic molecule to two near-resonance laser fields. Our study indicates that over a certain range of intensity of one of the fields, quenching of the absorption occurs. The quenching and the recovery have very sharp intensity thresholds. We studied the quenching and recovery thresholds as a function of the wavelength of the quenching radiation. The thresholds are found to exhibit oscillatory behavior as a function of the wavelength.

Laser-induced multiple excitation of molecules was previously treated using various models. ${ }^{1}$ One of the methods treats the excitation from the point of view of a one-dimensional nonrotating anharmonic oscillator model. In this respect, a harmonically driven anharmonic oscillator with a quartic anharmonicity describes excitation of vibrational levels in a molecule. We have used this model in the present study. The equation of motion of the oscillator can be shown to satisfy the following equation:

$$
\begin{aligned}
& {\left[\frac{d^{2}}{d t^{2}}+\epsilon \gamma \frac{d}{d t}+\omega_{0}^{2}-\epsilon d X^{2}\right) X} \\
& =\epsilon f_{1} \cos \left(\Omega_{1} t+v\right)+\epsilon f_{2} \cos \Omega_{2} t,
\end{aligned}
$$

where $\epsilon$ is a small parameter, $\omega_{0}$ is the resonant fequency of the harmonic oscillator, $\gamma$ is a damping coefficient, and $d$ is an anharmonicity parameter. The amplitudes $f_{1}$ and $f_{2}$ are equal to $\left(e_{\text {eff }} / M\right) E_{1}$ and $\left(e_{\text {eff }} / M\right) E_{2}$, respectively, where $M$ is the reduced mass of the vibrator, $E_{1}$ and $E_{2}$ are the amplitudes of excitation at frequencies $\Omega_{1}$ and $\Omega_{2}$, respectively, $v$ is some constant phase, and $e_{\text {eff }}$ is the classical effective charge which is related to the 
dipole matrix element $\mu$ by the relation

$$
e_{\text {eff }}=|\mu| \frac{\omega_{0}}{\hbar} M X_{0},
$$

where $X_{0}$ is the amplitude of oscillations at some arbitrary time. Equation (1) can be changed to nondimensional form by dividing it by $\omega_{0}^{2}$ and defining a nondimensional time $t^{\prime}=\omega_{0} t$, and a nondimensional amplitude $y=X / X_{0}$. We get

$$
\begin{aligned}
& \left(\frac{d^{2}}{d t^{\prime 2}}+\epsilon \gamma^{\prime} \frac{d}{d t^{\prime}}+1-\epsilon \alpha y^{2}\right) y \\
& =\epsilon F_{1}^{\prime} \cos \left(\omega_{1} t^{\prime}+v\right)+\epsilon F_{2}^{\prime} \cos \omega_{2} t,
\end{aligned}
$$

where $\quad \alpha=X_{0}^{2} d / \omega_{0}^{2}, \quad \omega_{1}=\Omega_{1} / \omega_{0}, \quad \omega_{2}=\Omega_{2} / \omega_{0}$, $\gamma^{\prime}=\gamma / \omega_{0}, F^{\prime}=|\mu| E_{1} / h \omega_{0}$, and $F_{2}^{\prime}=|\mu| E_{2} / h \omega_{0}$.

We are interested in the response of the oscillator when $\Omega_{1}$ and $\Omega_{2}$ are close to each other and in near resonance with the harmonic oscillator $\left(\omega_{1} \simeq \omega_{2} \sim 1\right)$. With the use of the slowly varying amplitude approximation, ${ }^{11}$ we write $y=A \cos \left(t^{\prime}+\beta\right)$ and $d y / d t=-A \sin \left(t^{\prime}+\beta\right)$, where $A$ and $\beta$ are slowly varying functions of time. Substituting these in Eq. (3), equating coefficients of $\sin \left(t^{\prime}+\beta\right)$ and $\cos \left(t^{\prime}+\beta\right)$, and neglecting the rapidly oscillating terms at frequencies $\left(1+\omega_{1}\right)$ and $\left(1+\omega_{2}\right)$, we get the following equations for $A$ and $\beta$ :

$$
\begin{gathered}
\frac{1}{\epsilon} \frac{d A}{d t}+\gamma^{\prime} A=-\frac{1}{2} F_{1}^{\prime} \sin \left[\left(1-\omega_{1}\right) t+\beta-v\right] \\
-\frac{1}{2} F_{2}^{\prime} \sin \left[\left(1-\omega_{2}\right) t+\beta\right], \\
\frac{A}{\epsilon} \frac{d \beta}{d t}=\frac{3}{8} \alpha A^{2}-\frac{1}{2} F_{1}^{\prime} \cos \left[\left(1-\omega_{1}\right) t+\beta-v\right] \\
-\frac{1}{2} F_{1}^{\prime} \cos \left[\left(1-\omega_{2}\right) t+\beta\right] .
\end{gathered}
$$

These equations can be written in a more convenient form by defining a new time scale $T=\alpha t$ :

$$
\begin{aligned}
\frac{1}{\epsilon} \frac{d A}{d T}+\gamma A= & \frac{1}{2} F_{1} \sin G \\
& +\frac{1}{2} F_{2} \sin \left[\left(\sigma_{2}-\sigma_{1}\right) \epsilon T+G-v\right], \\
\frac{A}{\epsilon} \frac{d G}{d T}= & \sigma_{1} A+\frac{3}{8} A^{3}+\frac{1}{2} F_{1} \cos G \\
& +\frac{1}{2} F_{2} \cos \left[\left(\sigma_{2}-\sigma_{1}\right) \epsilon T+G-v\right],
\end{aligned}
$$

where

$$
\begin{aligned}
& \gamma=\gamma^{\prime} / \alpha, \quad F_{1}=F_{1}^{\prime} / \alpha, \quad F_{2}=F_{2}^{\prime} / \alpha, \\
& \sigma_{1}=\left(\omega_{1}-1\right) / \epsilon \alpha, \quad \sigma_{2}=\left(\omega_{2}-1\right) / \epsilon \alpha,
\end{aligned}
$$

and

$$
G=\sigma_{1} \epsilon T-\beta+\nu .
$$

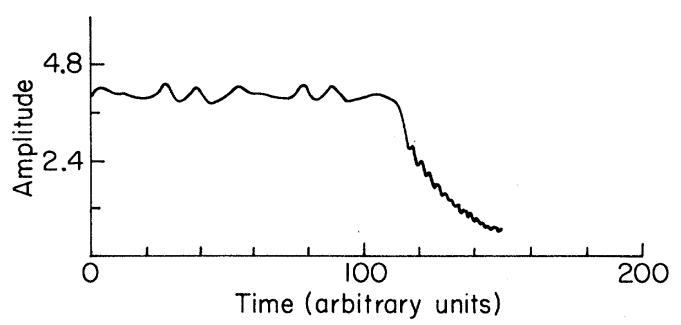

FIG. 1. Amplitude of the oscillator is plotted as a function of time for $F_{2}=0.215$ and $F_{1}=1$.

In the absence of the $F_{2}$ mode, the steady-state response can be derived analytically. After a sufficiently long time such that variation in the amplitude becomes negligible, then $d A / d T$ can be neglected, and consequently $d G / d T$ can also be neglected. In this limit we get

$$
\begin{aligned}
& \gamma A=\frac{1}{2} F_{1} \sin G, \\
& \sigma_{1} A=-\frac{3}{8} A^{3}-\frac{1}{2} F_{1} \cos G .
\end{aligned}
$$

Eliminating $G$ from Eqs. (8) and (9) gives the $\sigma_{1}-A$ response relation:

$$
\sigma_{1}=-\frac{3}{8} A^{2} \pm\left(\frac{F_{1}^{2}}{4 A^{2}}-\gamma^{2}\right)^{1 / 2} .
$$

This equation can also be alternatively written in the form

$$
A^{2}=\frac{F_{1}^{2}}{4\left[\left(\sigma_{1}+\frac{3}{8} A^{2}\right)^{2}+\gamma^{2}\right]}
$$

which shows that at optimal detuning $\sigma_{1}=-\frac{3}{8} A^{2}$, the oscillation has its maximum amplitude.

The effect of the second mode on the response is determined by integrating Eqs. (6) and (7) numerically. We examine in this study two cases. In the first case the $F_{1}$ mode is tuned such that, in the absence of the $F_{2}$ mode, it satisfies the above optimal detuning condition. In the second case, however, the $F_{1}$ mode is tuned such that, in the absence of the $F_{2}$ mode, it satisfies the steady-state response of Eq. (10). In the study we took $\epsilon=0.5, \sigma_{1}=-6$,

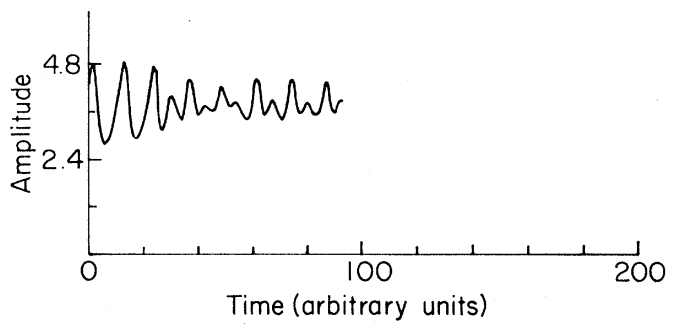

FIG. 2. Amplitude of the oscillator is plotted as a function of time for $F_{2}=2.3$ and $F_{1}=1$. 


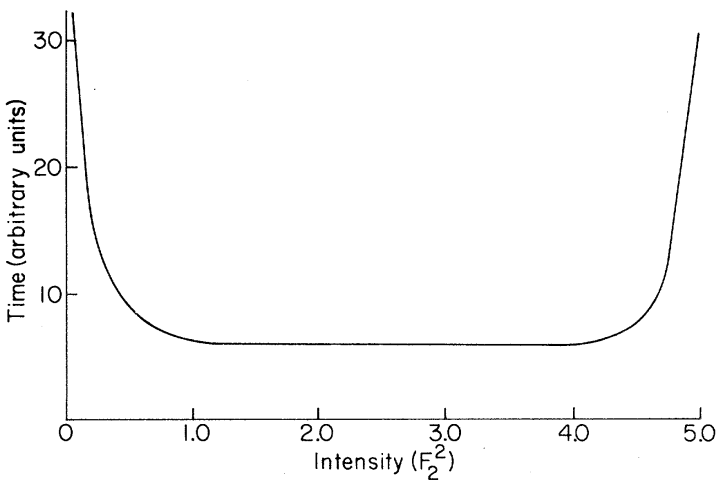

FIG. 3. Time during which the amplitude of the oscillator stays at its initial value as a function of $F_{2}^{2}$ for $F_{1}^{2}=1$.

$\sigma_{2}=-5, \quad F_{1}=1, \quad \gamma=0.1, \quad A(0)=4, \quad$ and $\beta(0)=0.9275$. We studied the response as a function of $F_{2}$. In the optimal detuning case, we found that for $F_{2}<0.215$, the system stays in its initial excitation. A very sharp threshold for quenching of the excitation, however, ocurrs at $F_{2}=0.215$. Figure 1 gives the amplitude of the excitation as a function of time for $F_{2}=0.215$ showing the quenching effect. This quenching of the excitation occurs with a decay rate given by the damping constant $\gamma=0.1$. As the intensity of $F_{2}$ increases further, a reversed threshold is encountered. At $F_{2} \simeq 2.3$, the system recovers from the state of no response as shown in Fig. 2. The calculation showed that there is a range in the amplitude of $F_{2}$ $\left(0.215<F_{2}<2.3\right)$ which results in quenching of the absorption. In Fig. 3 we plot the time during which the amplitude stays at its initial value as a function of $F_{2}^{2}$. Now keeping $A(0), \gamma$, and $F_{1}$ the same, but choosing $\sigma_{1}$ to be at exact resonance $\left(\sigma_{1}=-6.075\right)$ instead of the optimal detuning value $\sigma_{1}=-6$, we

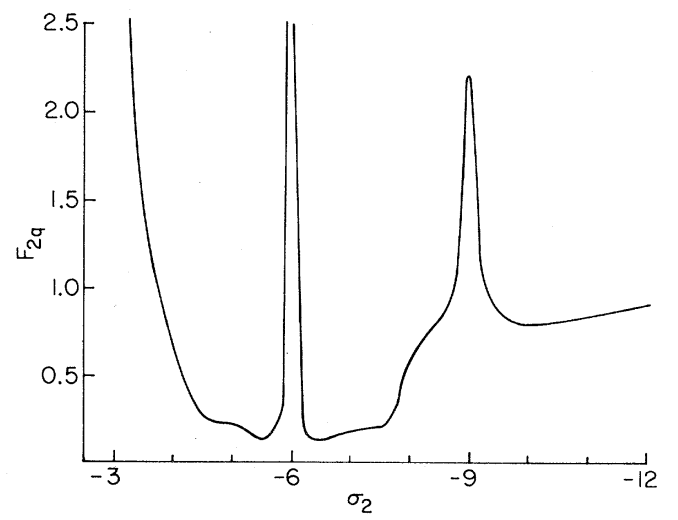

FIG. 4. Intensity threshold for quenching the absorption $F_{2 q}$, as a function of $\sigma_{2}$, the detuning of $F_{2}$ for the optimal detuning case.

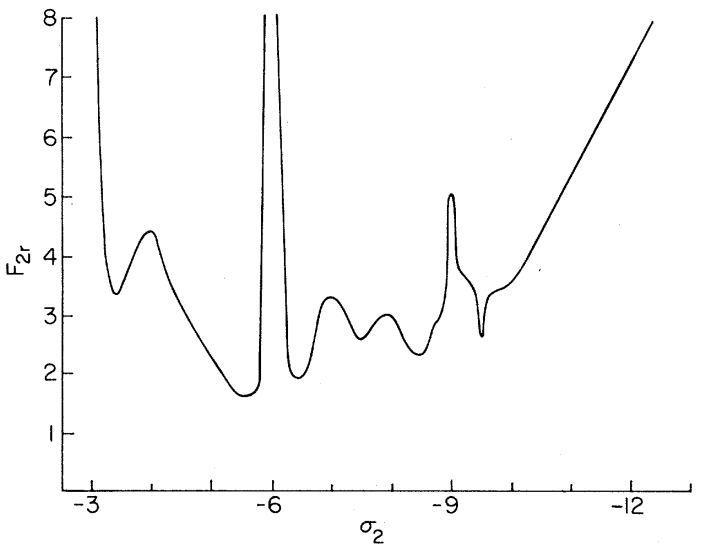

FIG. 5. Intensity threshold for the recovery from quenching $F_{2 r}$, as a function of $\sigma_{2}$, the detuning of $F_{2}$ for the optimal detuning case.

find that quenching occurs at a lower intensity $F_{2 q}=0.176$. However, recovery of the system occurred at $F_{2 r}=2.375$ which is slightly higher than the corresponding one of optimal detuning. As for the quenching window which occurs over a certain range in the intensity of the $F_{2}$ mode as shown in Fig. 3, the behavior of the system is very similar in both cases of the study.

It is interesting to examime the oscillatory behavior of the response. In the case of optimal detuning the steady-state response at small $F_{2}$ has $4 \%$ amplitude modulation at the $\sigma_{2}-\sigma_{1}$ difference frequency. The induced exponential decay of the initial amplitude has also $4 \%$ amplitude modulation but at a frequency equal to the detuning $\sigma_{1}$. In addition to this modulation the decay has a beat frequency at $\sigma_{1}-\sigma_{2}$. After the recovery the steadystate response has two trains of oscillations, each oscillating at the $\sigma_{2}-\sigma_{1}$ frequency. The trains have

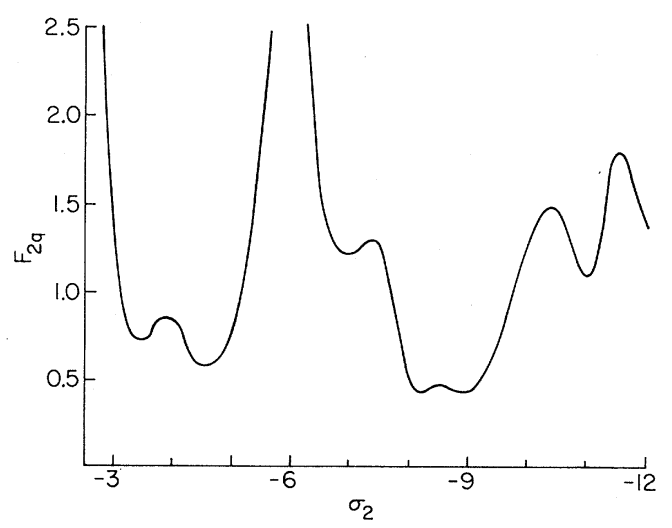

FIG. 6. Intensity threshold for quenching the absorption $F_{2 q}$, as a function of $\sigma_{2}$, the detuning of $F_{2}$ for the exact resonance case. 
different amplitudes and are phase shifted from each other. At higher $F_{2}$ intensities, i.e., $F_{2}=8$, the system goes back to a single-mode oscillation as in the case of small $F_{2}$ intensities.

We also studied the quenching effect as a function of the wavelength of the $F_{2}$ mode for optimal detuning. Figure 4 gives the intensity threshold for quenching the absorption, $F_{2 q}$, as a function of $\sigma_{2}$. We note that as $\sigma_{2}$ decreases starting from -3.5 , the threshold decreases sharply, becoming somewhat flat, and then tends towards larger and larger values as $\sigma_{2}$ approaches -6 . The difficulty in causing quenching at $\sigma_{2} \simeq-6$ is owing to the fact that the two modes become identical at this detuning. After crossing -6 , the threshold $F_{2 q}$ drops rapidly; as $\sigma_{2}$ moves away from -6 , it levels off and starts rising again. In the region around -9 it levels off and develops a local enhancement at $\sigma_{2}=-9$. This enhancement is due to the harmonic nature of this wavelength. Finally beyond this region it rises linearly.

Figure 5 gives the intensity threshold for the recovery from the quenching, $F_{2 r}$, as a function of $\sigma_{2}$ for the optimal detuning case. The figure shows oscillatory behavior in the region between $\sigma_{2}=-3.5$ and -9.5 . Beyond -9.5 , the recovery threshold rises linearly. Again as in the $F_{2 q}$ case, $F_{2 r}$ rises very steeply as $\sigma_{2}$ approaches -6 from either direction.

We have also studied the behavior of the oscillator at smaller damping, $\gamma=0.05$, under the influence of an $F_{1}$ mode of higher intensity $\left(F_{1}=2.52\right)$. We took $\epsilon=1, A(0)=4.1$, and $\sigma_{1}=-6$ satisfying the exact resonance condition [Eq. (10)]. We find behavior similar to that shown in Figs. 1-3. The $\sigma_{2}$ dependence of $F_{2 q}$ and $F_{2 r}$ for this case is shown in Figs. 6 and 7, respectively. The overall behavior, apart from some minute details, is similar to the op-

*Permanent address: Deparment of Physics, University of Jordan, Amman, Jordan.

${ }^{\dagger}$ Currently at Yarmouk University, Irbid, Jordan.

${ }^{1}$ See, for example, Multiphoton Processes, proceedings of the International Conference, University of Rochester, Rochester, New York, 1977, edited by J. H. Eberly and P. Lambprapolis (Wiley, New York, 1978).

${ }^{2} \mathrm{See}$, for example, Laser Applications To Chemistry, special Issue of the Optical Engineering Journal, edited by T. F. George, Opt. Eng. 19, No. 1 (1980).

${ }^{3}$ V. S. Letokhov, in Multiphoton Processes, Ref. 2, p. 331.

${ }^{4}$ R. V. Ambartzumian, Yu. A. Gorokhov, V. S. Letokhov, G. N. Markarov, A. A. Puretzky, and N. P. Furzikov, Zh. Eksp. Teor. Fiz. Pis'ma Red. 23, 217 (1976) [JETP Lett. 23, 194 (1976)]; R. V. Ambartzumian, N. P. Furzikov, Yu. A. Gorokhov, V. S. Letokhov, G. N.

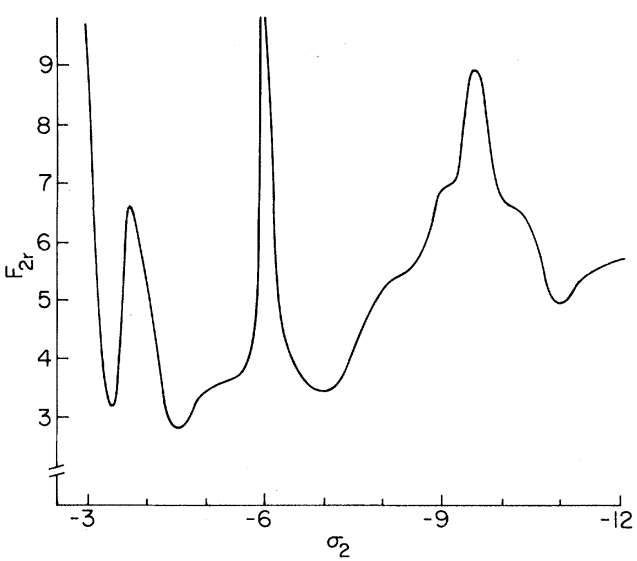

FIG. 7. Intensity threshold for the recovery from quenching $F_{2 r}$, as a function of $\sigma_{2}$, the detuning of $F_{2}$ for the exact resonance case.

timal detuning case. The obvious difference is that whereas the $\sigma_{2}$ dependence of $F_{2 q}$ is smooth and linear beyond $\sigma_{2}=-10$ for the optimal detuning case, it has an oscillatory nature in the exact resonance case. This situation is somewhat reversed in the $\sigma_{2}$ dependence of $F_{2 r}$ where the behavior is oscillatory in the region between $\sigma_{2}=-6$ and $\sigma_{2}=-9$ for the optimal detuning case, and somewhat smoothly increasing for the exact resonance case.

In conclusion, we have shown that the presence of certain intensities of an exciting mode can result in quenching of the molecular absorption induced by another mode. Further investigations gave the variation of the range of the quenching intensity with the wavelength of the exciting radiation.

This work was supported by National Science Foundation Grant No. NSF PHY 81-09305.

Markarov, and A. A. Puretzky, Opt. Commun. 18, 517 (1976).

${ }^{5}$ N. R. Isenor, V. Merchant, R. F. Hallsworth, and M. C. Richardson, Can. J. Phys. 51, 1281 (1973).

${ }^{6} \mathrm{~N}$. Boembergen, Opt. Commun. 15, 416 (1975).

7J. R. Stine and D. W. Noid, Opt. Commun. 31, 161 (1979).

${ }^{8}$ D. W. Noid, C. Bottcher, and M. L. Koszykowski, Chem. Phys. Lett. 72, 397 (1980).

9J. R. Stine and D. W. Noid, Chem. Phys. Lett. 77, 287 (1981).

${ }^{10}$ D. W. Noid and J. R. Stine, Opt. Commun. 37, 187 (1981).

${ }^{11}$ A. H. Nayfeh and D. Mook, Nonlinear Oscillations (Wiley-Interscience, New York, 1979). 